\title{
Varlitinib induced differential Protein Expression analysis on oral carcinoma cell line: A therapeutic approach
}

\section{Fariha Tanveer}

University of Karachi

\section{Amber llyas}

University of Karachi

\section{Basir Syed}

Chapman University

\section{Zehra Hashim}

University of Karachi

Aftab Ahmed

Chapman University

Shamshad Zarina ( $\square$ szarina@uok.edu.pk)

University of Karachi https://orcid.org/0000-0003-0781-7171

\section{Research Article}

Keywords: Proteomic analysis, varlitinib, mass spectrometry, signaling pathway, EGFR, OSCC

Posted Date: October 28th, 2021

DOI: https://doi.org/10.21203/rs.3.rs-1003477/v1

License: (1) This work is licensed under a Creative Commons Attribution 4.0 International License.

Read Full License 


\section{Abstract}

Receptor-ligand complex mediated signaling significantly contributesin cellular activities such as growth, proliferation, differentiation, and survival. However, augmented expression of signal transducing receptors and ligands is the most frequent molecular event and major hallmark of oral carcinogenesis. Among these receptors, Epidermal Growth Factor Receptor (EGFR) with intracellular tyrosine kinase activity is the most frequently overexpressed molecule by Squamous epithelial cells of oral cavity. Aberrated EGFR mediated signaling has laid the foundation of targeted therapy thus providing rationale for the conducted study. We have selected EGFR pathway as targeted intracellular signaling cascade inOral squamous cell carcinoma (OSCC). Deactivating EGFR by blocking the binding sites is likely to result in prevention of intracellular downstream signaling. In this context, Tyrosine Kinase Inhibitors (TKIs) have come into play. Quinazolines (aromatic heterocyclic compounds) and their derivatives have shown promising clinical outcomes. Present study focused to investigate anti-EGFR potential of quinazoline derivative, varlitinib-a pan-EGFR inhibitor on oral squamous epithelial cell lines. We performed proteomic analyses to identify differential expression pattern of proteins in SCC- 25 cells in response to varlitinib treatment. Identified proteins include Binding Immunoglobulin Protein (BiP), Heat Shock Protein 7C (HSP7C), Protein Disulfide Isomerase 1 A (PDIA1), Vimentin (VIME), Keratin type I Cytoskeletal 14 (K1C14), and $\beta$-Actin (ACTB). Among these, five proteinswere found to be downregulated upon varlitinib treatment whereas only Keratin type I Cytoskeletal 14 was upregulated. Differential expression of proteins and possible role of varlitinib as potential antitumor drug in oral carcinoma is discussed.

\section{Introduction:}

Oral squamous cell carcinoma (OSCC) constitutes a major proportionwith more than 100,000 cancer related fatalitiesworld-wide in 2020[1]. The pathophysiology of OSCC in part involves Epidermal Growth Factor Receptor (EGFR) and associated signaling pathway [2, 3]. EGFR is activated upon ATP mediated phosphorylation and subsequent receptor dimerization resulting in stimulation of pleiotropic downstream signaling cascade. These include Ras/Raf/MAPKand, PI3K/Akt, that control cell proliferation, differentiation, migration, and survival[4]. Dysregulation of EGFR and receptor mediated aberrated signaling cascade has been significantly involved in the development and progression of OSCC, resulting in invasion, metastasis, and angiogenesis [2-4].High expression of EGFR has been often associated with chemotherapeutic resistance towards the drugs being currently used in clinical settings such as methotrexate, 5 fluorouracil, cyclophosphamide etc. $[5,6]$. Other therapeutic strategies including radiotherapy have not gained substantial clinical outcomes for health improvement and overall survival [7]. These clinical findings draw attention towards pressing need to develop new, affective, and improved therapies for oral epithelial malignancy.

Studies based on signaling mechanisms led scientists to follow targeted therapeutic approach.Identification of EGFR as an oncogene has made EGFR pivotal drug target for the treatment of OSCC $[8,9]$. Compelling investigations on EGFR therapeutic ventureshave shown two main approaches 
targeting EGFR i.e., monoclonal antibodies (mAbs) and Tyrosine Kinase Inhibitors (TKIs). Each of this approach has distinct mechanism; mAbs (anti-EGFR antibodies) block the extracellular ligand binding domain whereas, TKIs target intracellular tyrosine kinase domain thus, preventing RTK activity [8]. Amongst TKIs, quinazolines have been significantly important.Quinazoline based drugs such as gefitinib, erlotinib, lapatinib, etc. are currently being clinically and pre-clinically tested for several epithelial malignancies including OSCC $[8,10]$

Varlitinib is an oral, pan, quinazoline based TK inhibitor of Human Epidermal Receptor (HER) with maximum efficacy in sub molar potency and minimal side effects[11]. Earlier, we reported anticancer effect of varlitinib on oral cancer cell line SCC-25 and identified that varlitinib mediates its action via MAPK/EGFR pathway [12]. Focus of current study was to investigate protein expression pattern in SCC25 squamous epithelial cell line in response to varlitinib treatment using proteomic approach.

\section{Materials And Methods:}

\subsection{Cell Culture and Drug Incubation:}

HumanSCC-25 cell line (ATCC® CRL-1628) Manassas, VA, USA) wascultured in Dulbecco's ModifiedEagle's Medium and Ham's F-12 Medium(DMEM/F-12). Mediumwas supplemented with 10\% heat-inactivated fetal bovine serum, $400 \mathrm{ng} / \mathrm{ml}$ hydrocortisone, $20 \mathrm{mmol} / \mathrm{L}$ glutamine, $100 \mathrm{U} / \mathrm{mL}$ penicillin and $100 \mu \mathrm{g} / \mathrm{mL}$ streptomycin. Cells were grown in humidified environment at $37^{\circ} \mathrm{C}$ and $5 \% \mathrm{CO} 2$. Later, they were sub-cultured in 1:4 ratio using $0.05 \%$ trypsin-EDTA and were seeded in 96-well microtiter plates (Corning, NY, USA) for the next $24 \mathrm{~h}$. The cells were then incubated with different concentrations of varlitinib $(1,5,10,20,30,40,50,60$ and $70 \mu \mathrm{M})$ for 24,48 , and $72 \mathrm{~h}$. Untreated SCC-25 cells incubated for similar time duration without drug were used as control.

\subsection{Cell Proliferation assessment:}

After varlitinib treatment,SCC- 25 cell viability was determined using MTS assay (Cell Proliferation assay kit, Abcam) as per manufacturer's instructions. Briefly, MTS reagent $(20 \mu \mathrm{l})$ was added in each well of 96well microtiter plate, followed by 2 hours incubation at $37^{\circ} \mathrm{C}$. The optical density of each well was then measured with a microplate reader (Beckman Coulter, California, USA) at $520 \mathrm{~nm}$. The percentage cytotoxicity was calculated as a growth percentage of cells relative to untreated controls. All assays were performed in triplicate.

\subsection{Two-Dimensional Gel Electrophoresis (2D-GE):}

Total protein was extracted from varlitinib treated and untreated SCC-25 cells using lysis buffer (Urea, Glycerol, NP-40, Ampholyte buffer 3-10, 0.5M Tris-HCL pH 6.8, 0.5M DTT, 0.25M EDTA). Total protein concentration in cell lysate was estimated using Pierce BCA Protein Assay Kit (Thermo Fisher Scientific, Waltham, MA, USA) as per manufacturer's protocol. 
$\sim 85 \mu \mathrm{g}$ protein from $50 \mu \mathrm{M}$ varlitinib treated cells for 24 hours and untreated cellswas dissolved in rehydration buffer containing $6 \mathrm{M}$ Urea, $2 \mathrm{M}$ Thiourea, $4 \% \mathrm{CHAPS}$, carrier ampholyte (Bio-Rad, Hercules CA, USA) and applied on immobilized pH-gradient (IPG) gel strips (7 cm. pH 3-10; Bio-Rad) at room temperature. The strips were rehydrated overnight. Subsequently, first dimension IEF was carried out using Multiphor II system (GE Health- care, England, UK) at $20^{\circ} \mathrm{C}$. The total voltage applied was 10,000 $\mathrm{V} /$ hwith constant current of $2 \mathrm{~mA}$. Following the IEF, focused strips were equilibrated in equilibration buffer A (6M Urea, $50 \mathrm{mM}$ Tris $\mathrm{pH} 8.8,30 \%$ Glycerol, $2 \%$ SDS with $10 \mathrm{mg} / \mathrm{ml}$ DTT) and buffer B (0.5M Tris$\mathrm{HCl} \mathrm{pH} 6.8,12 \mathrm{M}$ Urea, $10 \%$ SDS, $60 \%$ glycerol, $25 \mathrm{mg} / \mathrm{ml}$ lodoacetamide) for 30 minutes each. IPG strips were then loaded on to $12 \%$ SDS-Polyacrylamide gels. SDS-PAGE was run using Protean Cell system Bio Rad at constant voltage of 60 V. Protein spots were stained using Coomassie Brilliant Blue R-250.

\section{a) Image Analysis:}

Differential expression pattern of proteins from $50 \mu \mathrm{MVarlitinib}$ treated,and control gels were analyzed via PDQuest Gel Analysis Software Version 8.0.1 (Bio-Rad). We identified differentially expressed protein spots among control and treated gels. Spot intensities were calculated using PDQuest software followed by excision of differentially expressed spots via EXQuest spot cutter (BIO-RAD) for subsequent identification.

\subsection{MS/MS Analysis for protein identification:}

Differentially expressed protein spots were digestedusing trypsin. Briefly, protein spots were de-stained with $50 \mathrm{mM}$ Ammonium Bicarbonate, and $50 \%$ Acetonitrile (ACN) thrice for 15 minutes followed by dryingvia speed-vac concentrator (Eppendorf, Hamburg, Germany). Desiccated samples were rehydrated using $10 \mathrm{mM} \mathrm{DTT}$ and incubated for $30 \mathrm{~min}$ at $37^{\circ} \mathrm{C}$ to reduce disulfide linkages mediated by cysteine. Reduced cysteine residues were exposed with $50 \mathrm{mM}$ iodoacetamide in dark for 30 minutes at room temperature. Protein spots were enzymatically digested with $2 \mathrm{ng} / \mu \mathrm{L}$ of sequence grade trypsin (Promega). Resulting peptide fragments were obtained with $25 \mathrm{mM}$ Ammonium bicarbonate, $10 \%(\mathrm{v} / \mathrm{v})$ formic acid and acetonitrile (1:1) used in equal proportions. Subsequently, the peptide fragments were vacuum dried (Speed-Vacuum concentrator Eppendorf) and were re-suspended in $0.1 \%$ formic acid.For MS/MS analysis, peptides were subjected to Impact II UHR QqTOF, Bruker Mass Spectrometer. Protein identification was carried out using MGF files through Matrix Science search engine. Peptide fragmentation finger printing was opted for MASCOT search and the selected parameters were Carbamidomethyl (C) for fixed modification, Oxidation (M) for variable modification, maximum 2 missed cleavages were selected, peptide mass tolerance of $20 \mathrm{ppm}$ and fragment mass tolerance $0.5 \mathrm{Da}$ with $\mathrm{p}<$ 0.05 was selected. Protein identification of digested peptides was carried by using Swiss-Prot data base with MASCOT search engine.

\subsection{Bioinformatics:}

We analyzed PPI (protein-protein interaction) ofproteins identified in the current study using STRING (Search Tool for the Retrieval of Interacting Genes/Proteins) database with median confidence (scores greater than 0.4). 


\section{Results:}

\subsection{Cytotoxic potential of varlitinib on SCC-25 cells:}

Approximately 5000 cells were cultured in each well of 96 well plate. After 24 hours cells were incubated with different concentrations of varlitinib ( $\mu \mathrm{m}$ Conc.) for 24,48 and 72 hours. Cytotoxic activity of varlitinib on human oral epithelial SCC- 25 cellswas identified by MTS cell-proliferation assay.SCC-2 5 cells attained $50 \%$ cell growth inhibition (median dosage affecting $50 \%$ cell population) when exposed with 50 $\mu \mathrm{M}$ varlitinib for 24 hourswith respect to their control (Figure 1), $(p<0.05)$. We observed linear pattern of cytotoxicity in terms of dose and incubation time (Figure 2). We chose $50 \mu \mathrm{M}$ as cytotoxic dose for further proteomic analysis.

\subsection{Analysis of differential expression of proteins in human SCC-25 cell line:}

Comparison of protein expression levels in SCC-25 cell line treated with $50 \mu \mathrm{M}$ varlitinib for 24 hours was examined by treating cells along with respective control. Proteins extracted from treated and untreatedcells were subjected to 2D gel electrophoresis (2D-GE)to compare differentially expressed proteins. Maximum proteins were found in the pl range(s) of 4-7 and 7-8.5with molecular weight more than $25 \mathrm{kDa}$. Respective gel images of untreated control and $50 \mu \mathrm{M}$ varlitinib treated SCC- 25 cells are shown in Figure 3.

In this study, we identifiedsix differentially expressed spots via PDQuest softwarein a proteomic map of OSCC cells. The differential expression of these proteins was statistically significant (Figure 4) among control versus varlitinib treated gels. We found five proteins that happen to be downregulated in varlitinib treated SCC-25 cellswhereas oneprotein was found to be up regulated.

\subsection{Identification of differentially expressed proteins:}

Differentially expressed spots were submitted to Mass spectrometric analysis and the resulting data was analyzed using MASCOT protein identification search engine. A list of identified proteins with their molecular weight, pl, score, percent coverage and expression levels are given in Table 1. Relative spot intensities from spot 1 to spot 6 are given in Figure 4.

\subsection{Correlation of identified proteins with oral carcinogenesis:}

Interaction analyses of differentially expressed proteins identified via Mass spectrometric analyses were conducted using STRING network analysis tool.Based on protein interaction and pathway(s) data, obtained through STRING database and PubMed literature searches, we constructed a putative model of protein interaction networksinvolved in cellular regulating activities. Through experiments, databases, text 
mining and interaction with 2nd node methods in STRING, we found correlation of all differentially expressed proteins ACTB, K1C14, HSPs and VIME(Figure 5).

\section{Discussion:}

OSCC remains one of the deadliest malignancies in the past couple of decades possibly due to paucity of appropriate diagnostic and treatment modalities.Proteomic analyses with advances in mass spectrometry has paved the way by exploring novel biomarkers that could be used to timely and accurately diagnose OSCC. The aim of this study is to identify altered expression of proteins (that could be clinically useful) in OSCC upon varlitinib treatment. Cytotoxicity assessment of varlitinib showed dose and time dependent inhibitory action on cell proliferation. Growth inhibitory potential (in terms of reduced cell survival) was attained at 24 hours of treatment.

To determine the effects of varlitinib on protein expression levels in SCC-25 cells, we performed 2D-GE (two-dimensional gel electrophoresis) followed by Mass spectrometric analysis to identify differentially expressed proteins. The study exhibited 6 different proteinspots in total from varlitinib treated as well as control group of SCC- 25 cells.We observed significant fold changes in protein expression levels for 6 protein spotsafter 24 hours of treatment. The catalogue of these identified proteins using massspectrometry after treatment is shown in Table 1. Each protein is briefly described in the subsequent paragraphs.

We identified cytoskeletal protein (actin), extracellular matrix protein (keratin), heat shock proteins and molecular chaperones (Bip and HSP7C respectively).

The first group includes proteins that are correlated to evasion of apoptosis such asBiP, HSP7C and PDIA1. Binding immunoglobulin protein (BiP, also known as Glucose Regulated Protein-78; GRP-78) is a member of HSP 70 family hence encoded by HSPA5 gene [13]. BiP is an Endoplasmic Reticulum (ER) residing molecular chaperone protein. It is responsible for mediating folding, translocation of proteins, initiating unfolded protein response (UPR) and ER-associated degradation (ERAD).BiP is of utmost importance for regulating homeostasis[13]. Under stress condition(s), BiP undergoes several changes in terms of function, expression and activation which blocks translocation of proteins to ER. Subsequently causing impaired degradation resulting in pathological conditions such as cancers, cardiovascular as well as neurodegenerative disorders [13]. BiP is an established master regulator of cancer and has been found to be over-expressed in wide variety of cancers including lung cancer, liver cancer, breast cancer etc. $[13,14]$. Increased expression of BiP inactivatespro-apoptotic markers such as BIK and BAX andinhibits apoptosis $[13,15]$. BiP, through signal transducer, activates intracellular kinases thus activating cellular pathways that promote cell proliferation and survival [16]. Moreover, BiP plays a critical role in OSCC progression by inducing enhanced proliferation, chemoresistance and metastasis[17].BiP implicitly activates MAPKand PI3Ksignaling cascades facilitating cancer cell progression. [18]. BiP pathogenesis is evidenced through different studies [16-19]. It has been suggested that downregulation of BiP can be potentially linked with impeded tumor formation and growth resulting in apoptotic 
induction and improved survival. Our study shows down-regulation of $\mathrm{BiP}$ in response to varlitinib treatment suggesting therapeutic potential of the drug.

Protein disulfide isomerase 1 (PDIA1) - a dithiol-disulfide oxidoreductase is also an ER residing molecular chaperone [20, 21]. PDIA1 primarily catalyzes disulfide bond formation via its oxidizing, reducing and isomerizing capabilities thusmediating proteinfolding, translocation to ER and degradation thus contributing to maintain cellular homeostasis[20, 21]. Deregulated PDIA1 expression and/or enzyme activity is often associated with different human diseases such as CVDs, neurodegenerative disorders, and different cancer types [21]. Higher expressions of PDIA1 have been demonstrated in brain cancer [22], lymphoma [23], colorectal cancer[24], breast cancer [25], often conferring metastasis, invasiveness and chemoresistance. The mechanisms causing increased expression of PDIA1 and associated pathological outcomes are poorly understood [24]. However, PDIA1 is considered as an upstream regulator for balancing Reactive Oxygen Species (ROS) as well as controlling the activity of a metalloprotease ADAM17 which in turn acts as an intermediate in EGFR signaling[25]. ADAM17 activation is based on change in redox balance - an effect which is important for growth factor dependent signaling in cancer cells. It is reported that gene silencing or knocking out PDIA1 results in induction of apoptosis and decreased cell proliferation [24]). Ourproteomics data revealed upregulation of PDIA1 in control SCC-25 cellswhile varlitinib treated group showed reduction in PDIA1 expression levels. The results remainedconsistent with previously conducted studies.

Another interesting protein found to be decreased in varlitinib treated cells was HSP7C. HSP7C is a 71 KDaheat shock related proteinwhich is constitutively expressed in cells and regulates trafficking and folding of nascent proteins. Ithas been frequently observed in human breast cancer [26] autoimmune retinopathy [27] etc.Increased expression of HSP7C due to heat, oxidative stress and hypoxia promotes cell survival, apoptotic evasion from proteolytic stress related rapid and abnormal cell growth[28]. Our proteomics data showed increased expression of HSP7C among SCC-25 cells which is in consensus with the already performed studies $[28,29]$. The expression was found to be decreased following 24 hours varlitinib treatment of SCC- 25 cells confirming its oncogenic role.

The second group of proteins belong to the family of intermediate filament proteins including Vimentin and cytoskeletal Keratin Type 14. Vimentin plays key role in cellular, physiological,structural, mechanical, biological, and developmental processes. Vimentin is known to provide phosphorylation site(s) for kinases thereby regulating signal transduction[30]. Vimentin has been found to be present in wide range of cells including mesenchymal cells and is typically associated with Epithelial to mesenchymal transition - a prominent feature associated with tumor progression [31]. Increased vimentin expression is often associated with breast cancers [31], gastrointestinal [31] and prostate cancer [32].Knock down clones of these proteins have shown decline in carcinoma cell proliferation and decreased vimentin expression could likely lead to anti-neoplastic behavior of cells by driving them less aggressive and controlled proliferation [33-35]. We also observed reduced expression of vimentin in varlitinib treated SCC-25 cell line in comparison to control cells. Second protein, Keratin is also a member of the family of intermediate filament and is reported as protein marker of epithelial differentiation generating polarity 
which plays a significantly importantrole in providing mechanical strength and integrity to epithelial cells $[36,37]$ Besides mechanical support and stability, keratins contribute in intracellular signal transduction $[36,37]$. We identified Type I Keratin cytoskeletal 14 which is typically expressed in basal layer cells of non-keratinized stratified squamous epithelial lining [36]. K1C14 has been associated with carcinogenesis and it is usually overexpressed in different cancers[37, 38]. However, we found up-regulated expression of K1C14 among varlitinib treated group of SCC- 25 cellswith respect to their counter control. Our results are contradictory to previous reports and needs to be explored further to identify the exact pattern of K1C14 in response to TKI mediated treatment.A possible reason for thiscontradiction in expression pattern of keratins could be modified expression of genes and proteins (such as cell cycle regulator biomarker p21), associated with carcinogenesis[36]. This, in turn, may result in responding differently to varlitinib mediated targeted therapy. However, alterations at genetic or protein level are key players behind progression / pathogenesis of oral carcinogenesis [36] resulting in apoptotic resistance [38, 39].

Lastly, ACTB Human Actin, cytoplasmic 1 is one of the six isoforms of actin protein, which is considered a highly conserved and ubiquitously expressed [40] protein. Actin is essentially involved in cellular activities such as motility, development etc.[40].Actin has integral part in survival for most of the cells since it provides mechanical support and force to drive cellular activities as well as motility. Cancer cells also undergo locomotion by moving from one location in the body to another via making contacts with neighboring cells[41], resulting in migration. ACTB has been found to be upregulated during different cancers including prostate, breast, and colorectal cancers [42].In the present study, ACTB showedincreased expressionin control SCC-25 cells whereas $50 \mu \mathrm{M} 24$ hours prolonged treatment resulted in downregulation of ACTB. This agreeswith previous reports [43, 44]. Protein-protein interaction via STRING 9.1 with experimental databases (including GO, KEGG etc.) and text-mining as active methods of predicting protein associations indicated that all identified proteins interacted with each with an exception of KR1T14.

\section{Conclusion:}

Thisstudy focused on varlitinib mediated differential expression of proteins involved in EGFR signaling cascade in SCC-25 cells. Our dataindicated that varlitinib might be considered as a potenttherapeutic agent with promising anti-proliferative capabilityconcomitantlyresulting in suppression of anti-apoptotic proteinsresulting in cessation of oral carcinogenesis.

\section{Declarations}

\section{Funding}

Partial funding by Dean Science Grant, University of Karachi, Karachi

\section{Conflict of Interest}

Authors declare that there is no conflict of interest 


\section{Data Availability}

On request

\section{Consent for Publication}

All authors agree with publication

\section{Author Contribution}

FT performed bench work and wrote the draft, Al contributed in cell culture studies, ZH conducted in-gel digestion and two D electrophoresis, BS performed MS analysis, AA and ZH did data interpretation, SZ conceived the project, supervised the study and finalized the manuscript.

\section{Code Availability}

Not Applicable

\section{Cell lines}

HumanSCC-25 cell line (ATCC® CRL-1628) Manassas, VA, USA)

\section{References}

1. Sung H, Ferlay J, Siegel RL, Laversanne M, Soerjomataram I, Jemal A, Bray F (2021) Global cancer statistics 2020: GLOBOCAN estimates of incidence and mortality worldwide for 36 cancers in 185 countries. CA: a cancer journal for clinicians 71:209-249

2. Grandis JR, Tweardy DJ TGF-a and EGFR in head and neck cancer (1993). Journal of Cellular Biochemistry. 53:188-191

3. Wong DTW, Todd R, Tsuji T, Donoff RB (1996) Molecular biology of human oral cancer. Critical Reviews in Oral Biology Medicine 7:319-328

4. Salomon DS, Brandt R, Ciardiello F, Normanno N (1995) Epidermal growth factor-related peptides and their receptors in human malignancies. Critical reviews in oncology/hematology 19:183-232

5. Lagha A, Chraiet N, Ayadi M, Krimi S, Allani B, Rifi H, Raies H, Mezlini A (2012) Systemic therapy in the management of metastatic or advanced salivary gland cancers. Head Neck Oncology 4:1-12

6. Zheng HC. The molecular mechanisms of chemoresistance in cancers.(2017) Oncotarget. 8:59950

7. Kim SH, Lee WH, Kim SW, Je HU, Lee JC, Chang HW, Kim YM, Kim K, Kim SY, Han MW (2018) )EphA3 maintains radioresistance in head and neck cancers through epithelial mesenchymal transition. Cellular signaling 47:122-130

8. Sabbah DA, Hajjo R, Sweidan K (2020) Review on epidermal growth factor receptor (EGFR) structure, signaling pathways, interactions, and recent updates of EGFR inhibitors. Current topics in medicinal chemistry 20:1-20 
9. Byeon HK, Ku M, Yang J (2019) Beyond EGFR inhibition: multilateral combat strategies to stop the progression of head and neck cancer. Exp Mol Med 51:1-14

10. Takei J, Kaneko MK, Ohishi T, Kawada M, Harada H, Kato Y (2020) A novel antiEGFR monoclonal antibody (EMab17) exerts antitumor activity against oral squamous cell carcinomas via antibodydependent cellular cytotoxicity and complementdependent cytotoxicity. Oncology letters 19:2809-2816

11. Gras J (2018) Varlitinib tosylate. Pan-HER tyrosine kinase inhibitor, Treatment of cancer. Drugs of the future 43:815-822

12. Usman M, Tanveer F, llyas A, Zarina S (2020) Varlitinib Mediates Its Activity Through Down Regulating MAPK/EGFR Pathway in Oral Cancer. Current Proteomics 17:51-58

13. Wang J, Lee J, Liem D, Ping P (2017) HSPA5 Gene encoding Hsp70 chaperone BiP in the endoplasmic reticulum. Gene 618:14-23

14. Shnyder SD, Mangum JE, Hubbard MJ (2008) Triplex profiling of functionally distinct chaperones (ERp29/PDI/BiP) reveals marked heterogeneity of the endoplasmic reticulum proteome in cancer. $\mathrm{J}$ Proteome Res 7:3364-3372

15. Luo B, Lee AS (2013) The critical roles of endoplasmic reticulum chaperones and unfolded protein response in tumorigenesis and anticancer therapies. Oncogene 32:805-818

16. Motaung B, WalzI G, Loxton AG (2019) The level of the endoplasmic reticulum stress chaperone protein, binding immunoglobulin protein (BiP), decreases following successful tuberculosis treatment. International Journal of Infectious Diseases 81:198-202

17. Garshott DM, Bechler SA, Burchhardt DM, O’Brien PS, Yoo GH, Chiego DJ, Rehman AO, Callaghan MU, Fribley AM (2016) The unfolded protein response as a therapeutic target for head and neck squamous cell Carcinoma. InTargeting Oral Cancer. Springer, Cham.. pp. 225-261

18. Schwarze S, Rangnekar V (2010) Targeting plasma membrane GRP78 for cancer growth inhibition. Cancer Biol Ther 9:153-155

19. Ruggiero C, Doghman-Bouguerra M, Ronco C, Benhida R, Rocchi S, Lalli E(2018) The GRP78/BiP inhibitor HA15 synergizes with mitotane action against adrenocortical carcinoma cells through convergent activation of ER stress pathways. Molecular and cellular endocrinology. 474;57-64

20. Ferrari DM, SÖLING HD (1999) The protein disulphide-isomerase family: unravelling a string of folds. Biochemical Journal 339:1-10

21. Xu S, Sankar S, Neamati N (2014) Protein disulfide isomerase: a promising target for cancer therapy. Drug discovery today 19:222-240

22. Rickman DS, Bobek MP, Misek DE, Kuick R, Blaivas M, Kurnit DM, Taylor J, Hanash SM (2001) Distinctive molecular profiles of high-grade and low-grade gliomas based on oligonucleotide microarray analysis. Cancer research 61:6885-6891

23. Piccaluga PP, Agostinelli C, Califano A, Rossi M, Basso K, Zupo S, Went P, Klein U, Zinzani PL, Baccarani M, Facera RD, Pileri SA (2007) Gene expression analysis of peripheral T cell lymphoma, 
unspecified, reveals distinct profiles and new potential therapeutic targets. J Clin Investig 117:823834

24. De Bessa TC, Pagano A, Moretti AIS, Oliveira PVS, Mendonça SA, Kovacic H, Laurindo FRM (2019) Subverted regulation of Nox1 NADPH oxidase-dependent oxidant generation by protein disulfide isomerase A1 in colon carcinoma cells with overactivated KRas. Cell death disease 10:1-16

25. Chahed K, Kabbage M, Ehret-Sabatier L, Lemaitre-Guillier C, Remadi S, Hoebeke J, Chouchane L (2005) Expression of fibrinogen E-fragment and fibrin E-fragment is inhibited in the human infiltrating ductal carcinoma of the breast: the two-dimensional electrophoresis and MALDI-TOFmass spectrometry analyses. Int J Oncol 27:1425-1431

26. Lee E (2017) Emerging roles of protein disulfide isomerase in cancer. BMB Rep 50:401

27. Fujioka H, Sakai A, Tanaka S, Kimura K, Miyamoto A, Iwamoto M, Uchiyama K (2017) Comparative proteomic analysis of paclitaxel resistancerelated proteins in human breast cancer cell lines. Oncology letters 13:289-295

28. Adamus G, Bonnah R, Brown L, David L (2013) Detection of autoantibodies against heat shock proteins and collapsin response mediator proteins in autoimmune retinopathy. BMC Ophthalmol 13:1-9

29. Murphy ME (2013) The HSP70 family and cancer. Carcinogenesis 34:1181-1188

30. Galli M, Pagni F, De Sio G, Smith A, Chinello C, Stella M, L'Imperio V, Manzoni M, Garancini M, Massimini D, Mosele N (2017) Proteomic profiles of thyroid tumors by mass spectrometry-imaging on tissue microarrays. Biochimica et Biophysica Acta (BBA)-Proteins and Proteomics. 1865:817-27

31. Arun S, Shulin L (2011) Vimentin as a potential molecular target in cancer therapy Or Vimentin, an overview and its potential as a molecular target for cancer therapy. Cell Mol Life Sci 68:3033-3046

32. Wei, J., Xu, G., Wu, M., Zhang, Y., Li, Q., Liu, P... Ma, D.(2008) Overexpression of vimentin contributes to prostate cancer invasion and metastasis via src regulation. Anticancer research. 28:327-334

33. Alam H, Sehgal L, Kundu ST, Dalal SN, Vaidya MM (2011) Novel function of keratins 5 and 14 in proliferation and differentiation of stratified epithelial cells. Molecular biology of the cell 22:40684078

34. Liu X, Wu Y, Zehner ZE, Jackson-Cook C, Ware JL (2003) Proteomic analysis of the tumorigenic human prostate cell line M12 after microcell-mediated transfer of chromosome 19 demonstrates reduction of vimentin. Electrophoresis 24:3445-3453

35. Yi W, Peng J, Zhang Y, Fu F, Zou Q, Tang Y (2013) Differential protein expressions in breast cancer between drug sensitive tissues and drug resistant tissues. Gland surgery 2:62

36. Moll R, Divo M, Langbein L (2008) The human keratins: biology and pathology. Histochemistry cell biology 129:705

37. Frohwitter G, Buerger H, Van Diest PJ, Korsching E, Kleinheinz J, Fillies T (2016) Cytokeratin and protein expression patterns in squamous cell carcinoma of the oral cavity provide evidence for two distinct pathogenetic pathways. Oncology letters 12:107-113 
38. Streckfus C (2016)Altered Salivary Protein Profiles among Individuals Diagnosed with Cervical Dysplasia and Cervical Carcinoma in Situ. Cancer and clinical oncology. 5:63-72

39. Vaidya MM, Kanojia D (2007) Keratins: markers of cell differentiation or regulators of cell differentiation? Journal of biosciences 32:629-634

40. Patrinostro X, Roy P, Lindsay A, Chamberlain CM, Sundby LJ, Starker CG, Voytas DF, Ervasti JM, Perrin BJ. (2018)Essential nucleotide-and protein-dependent functions of Actb/ $\beta$-actin. Proceedings of the National Academy of Sciences. 115:7973-8

41. Witjes L, Van Troys M, Verhasselt B, Ampe C (2020) Prevalence of Cytoplasmic Actin Mutations in Diffuse Large B-Cell Lymphoma and Multiple Myeloma: A Functional Assessment Based on Actin Three-Dimensional Structures. Int J Mol Sci 21:3093

42. Khimani AH, Mhashilkar AM, Mikulskis A, O'Malley M, Liao J, Golenko EE, Mayer P, Chada S, Killian JB, Lott ST (2005) Housekeeping genes in cancer: normalization of array data. Biotechniques 38:739-745

43. Chang TJ, Juan CC, Yin PH, Chi CW, Tsay HJ (1998) Up-regulation of beta-actin, cyclophilin and GAPDH in N1S1 rat hepatoma. Oncol Rep 5:469-471

44. Goh WWB, Lee YH, Ramdzan ZM, Chung MC, Wong L, Sergot MJ (2012) A network-based maximum link approach towards MS identifies potentially important roles for undetected ARRB1/2 and ACTB in liver cancer progression. International journal of bioinformatics research applications 8:155-170

\section{Figures}

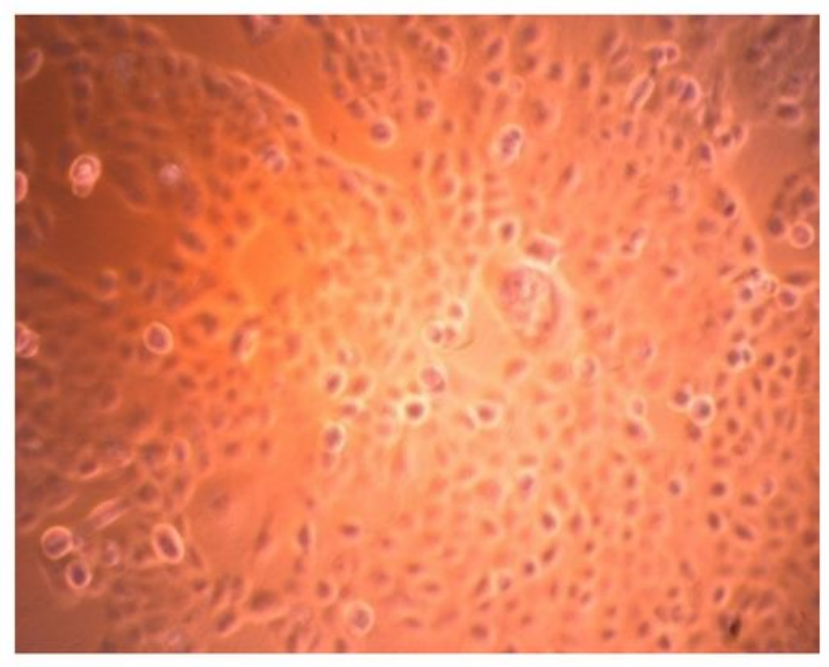

a

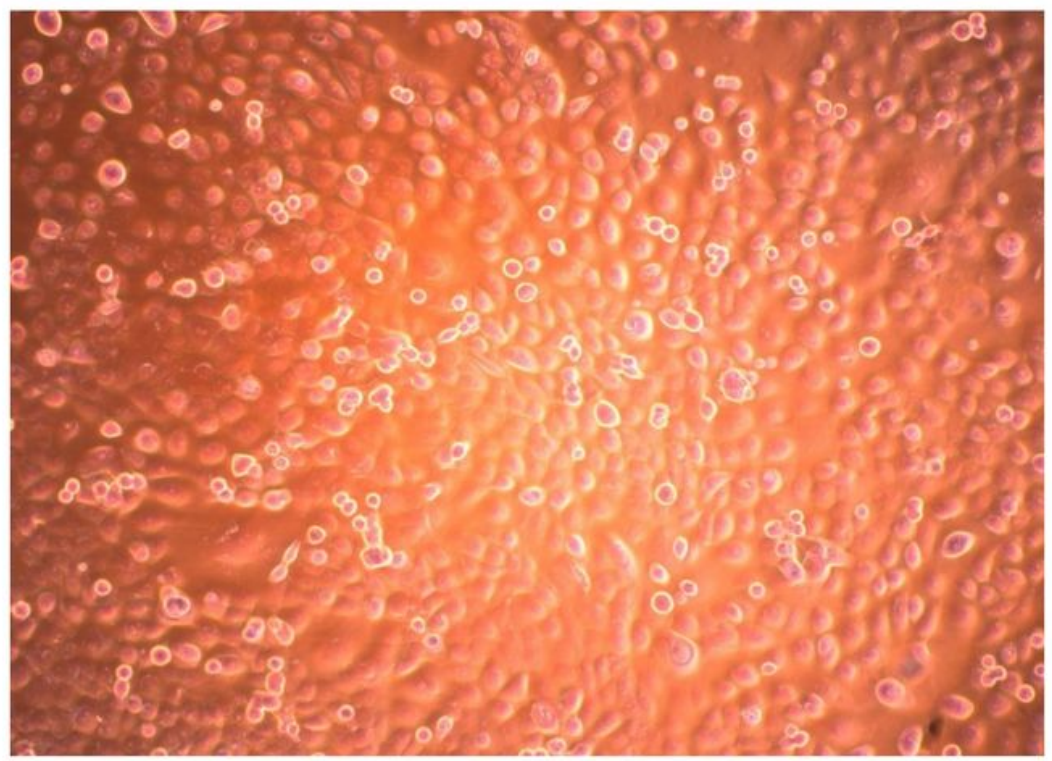

\section{Figure 1}

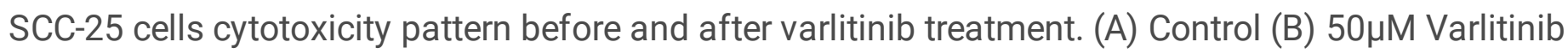
treatment for 24 hours. 


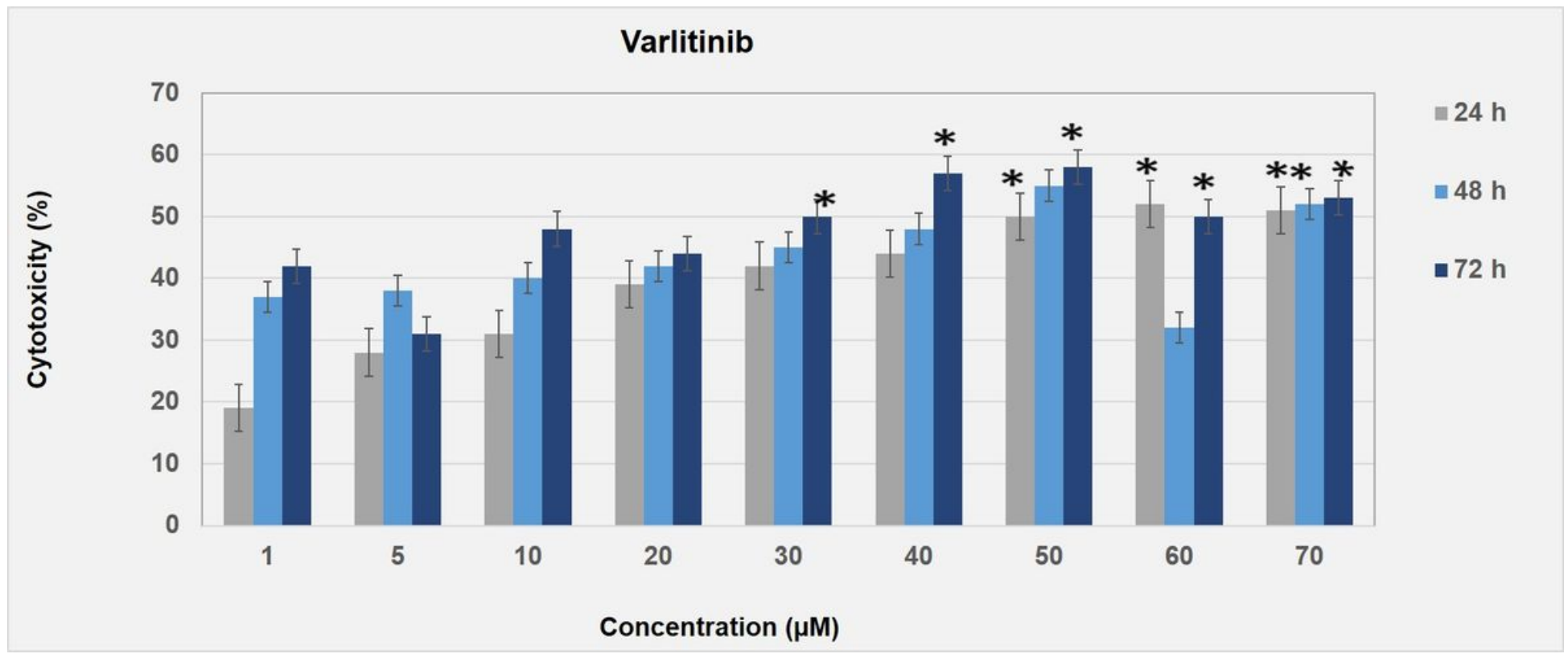

\section{Figure 2}

The effect of different concentrations of Varlitinib $(1 \mu \mathrm{M}, 5 \mu \mathrm{M}, 10 \mu \mathrm{M}, 20 \mu \mathrm{M}, 30 \mu \mathrm{M}, 40 \mu \mathrm{M}, 50 \mu \mathrm{M}, 60$ $\mu \mathrm{M}$ and $70 \mu \mathrm{M}$ ) on SCC-25 cells after 24-, 48- and 72-hours treatment. Cytotoxicity was determined by MTS assay. Each bar represents mean percent cytotoxicity \pm S.D. for each concentration respectively. Data sets were analysed for statistically significant differences by one way ANOVA. * represents statistically significant difference $(P<0.05)$ between treated and untreated cells.
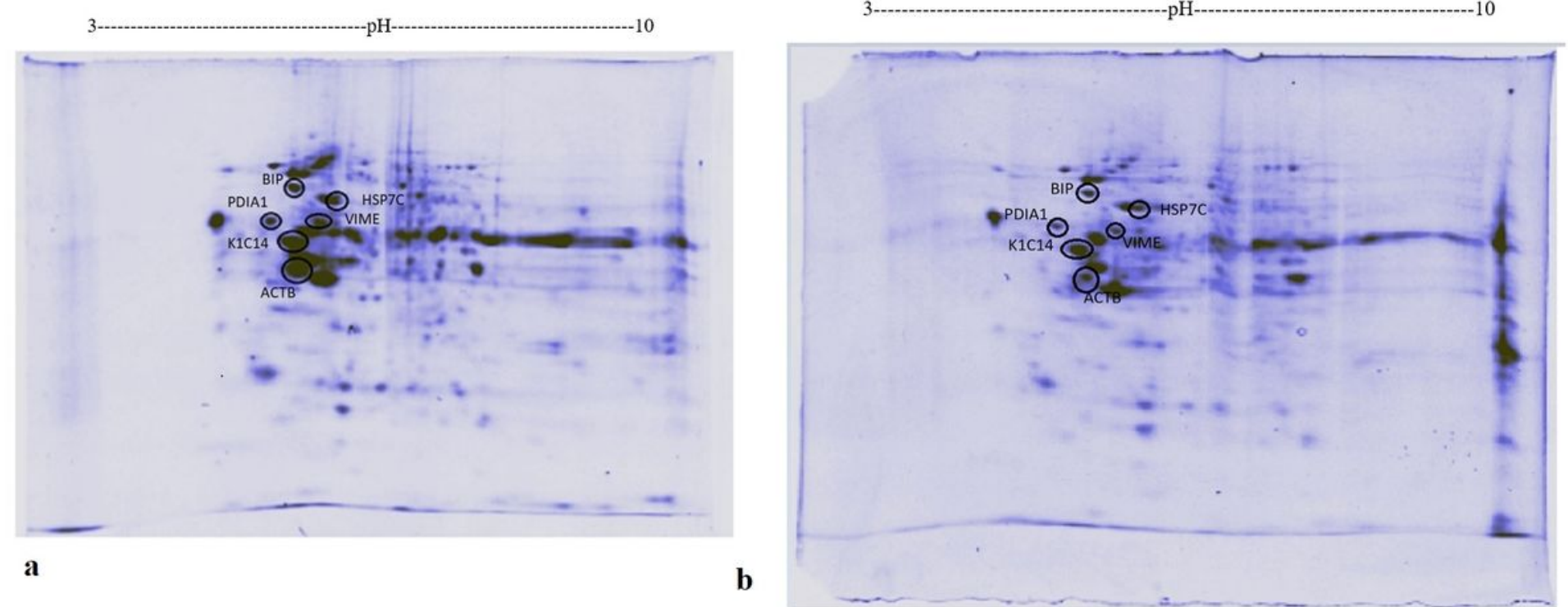

\section{Figure 3}

Representative images of two-Dimensional gel electrophoresis from untreated control (A) and $50 \mu \mathrm{M}$ varlitinib treated (B) SCC-25 cells using Immobilized pH Gradient (IPG) strips 3-10 NL. Identified protein spots are encircled and labelled. 

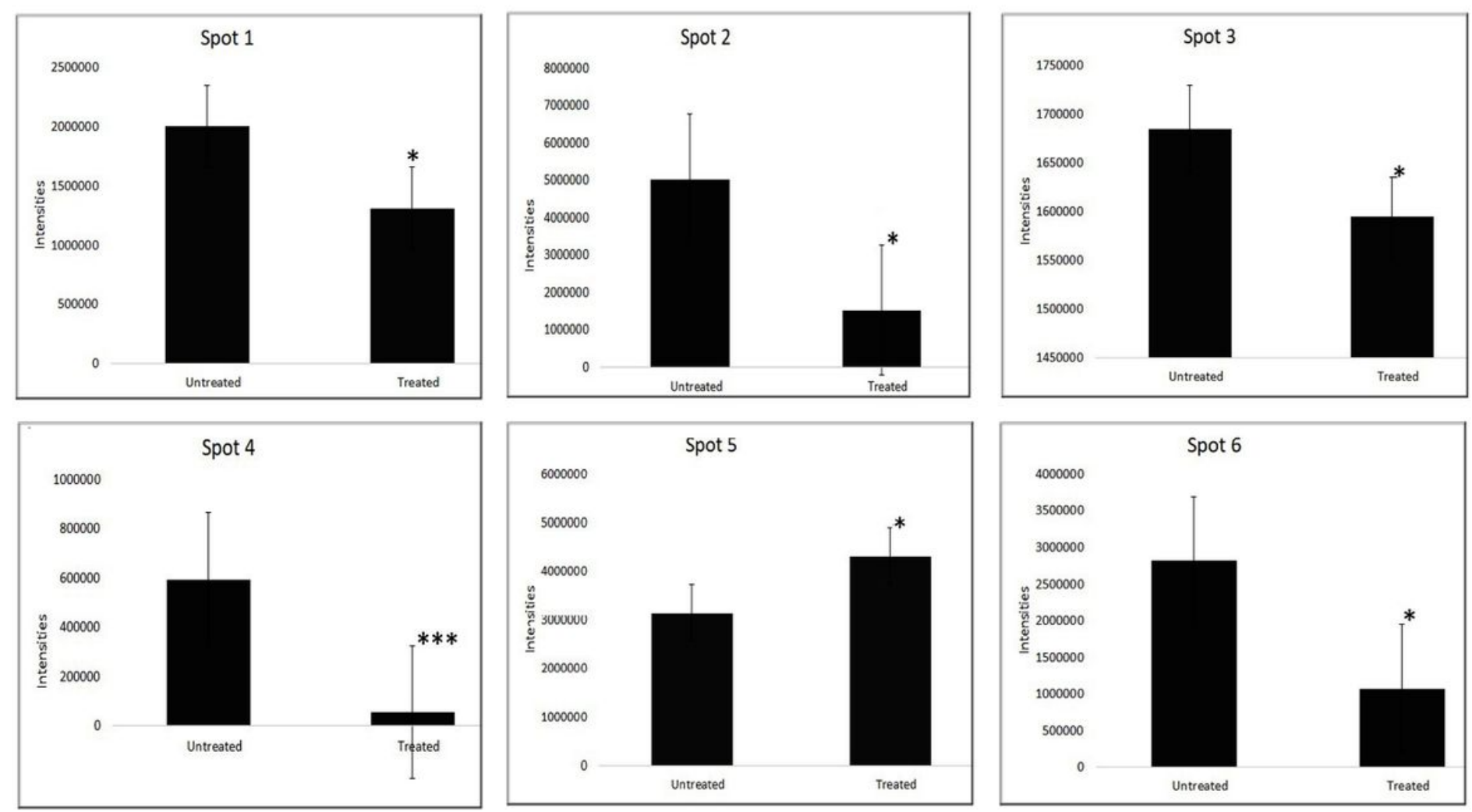

Figure 4

Spot intensities of differentially expressed identified proteins from untreated and varlitinib treated SCC-25 cells. Error bars indicate \pm SD whereas * indicates statistical significance $(p<0.05)$.

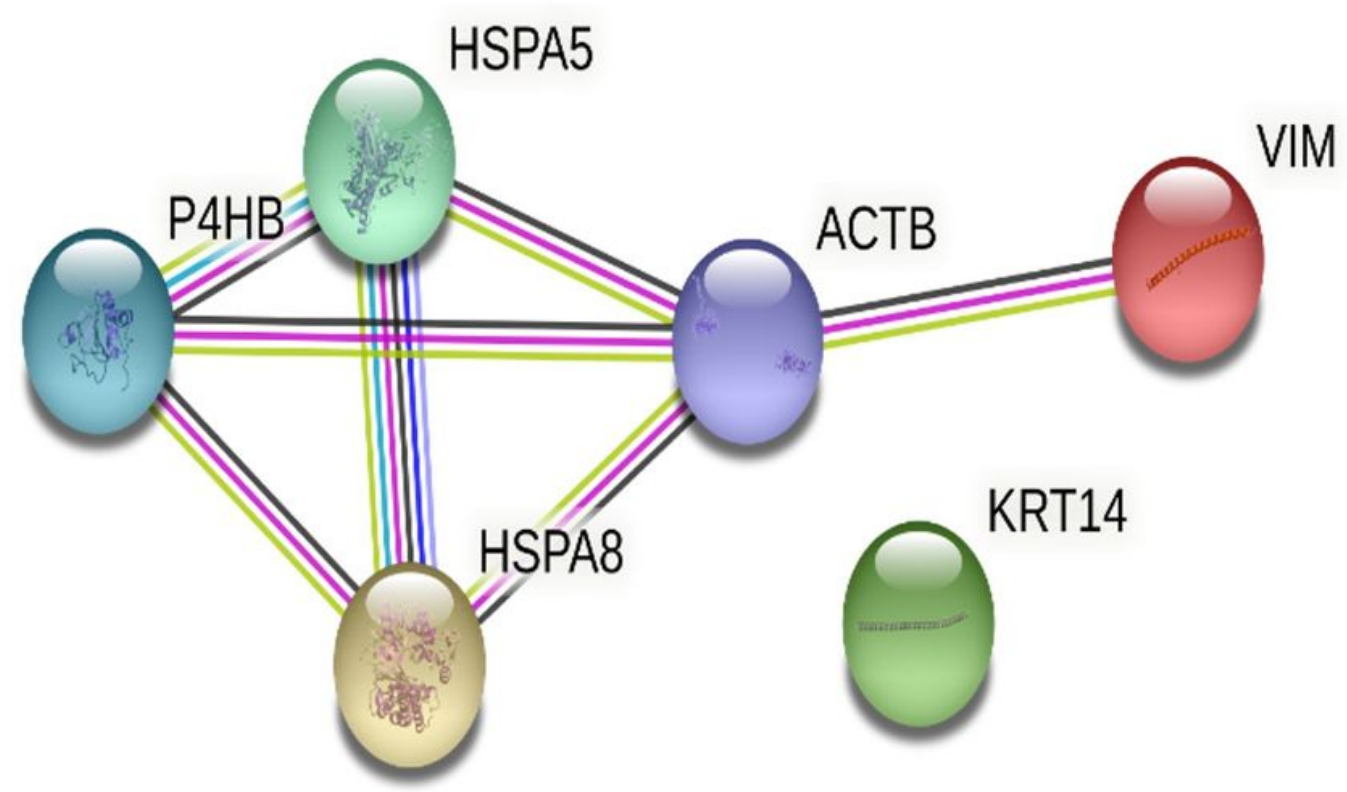

Figure 5 
Protein-Protein interaction analysis of identified proteins as observed through STRING. Identified proteins include PH4B (PDIA1); Protein di-sulfide isomerase 1, KRT14; Keratin type 14, VIM; Vimentin, HSPA8; Heat shock cognate $71 \mathrm{kDa}$ protein, HSPA5; Endoplasmic reticulum chaperone BiP and ACTB; Beta actin. Nodes represent proteins while edges show protein association. 